\title{
Nanocrystalline Phase Formation inside Shear Bands of Pd-Cu-Si Metallic Glass
}

\author{
Yang Shao,,$^{1,2}$ Guannan Yang, ${ }^{1,2}$ and Kefu Yao ${ }^{1,2}$ \\ ${ }^{1}$ School of Materials Science and Engineering, Tsinghua University, Beijing 100084, China \\ ${ }^{2}$ Key Laboratory for Advanced Materials Processing Technology, Ministry of Education, Tsinghua University, Beijing 100084, China
}

Correspondence should be addressed to Yang Shao; shaoyang@tsinghua.edu.cn

Received 21 March 2014; Accepted 23 April 2014; Published 14 May 2014

Academic Editor: Na Chen

Copyright (c) 2014 Yang Shao et al. This is an open access article distributed under the Creative Commons Attribution License, which permits unrestricted use, distribution, and reproduction in any medium, provided the original work is properly cited.

$\mathrm{Pd}_{77.5} \mathrm{Cu}_{6} \mathrm{Si}_{16.5}$ metallic glass was prepared by fluxing treatment and water quenching method. To avoid possible artifacts, shear bands were created by indentation after TEM sample preparation. Bright field image, diffraction pattern, and the dark field image of TEM that covered the shear band region were presented. A few nanocrystalline phases were noticed inside the shear bands, which favored the plastic deformation ability and supported the explanation of mechanical deformation-induced crystallization.

\section{Introduction}

Metallic glasses (MGs) are a kind of advanced engineering materials due to their high strength, high elastic limit, and better corrosion resistance compared to their crystalline counterparts [1]. However, two dilemmas apparently deteriorate MGs' applications as promising structural engineering materials: one is the limited critical size, and the other is the poor plastic deformation ability for most MGs. The plastic carriers of MGs, shear bands, are of two-dimensional defects. Their formation and propagation are fundamentally important for understanding the plastic deformation mechanism in MGs. However, little is known so far and even the structure of shear band is still a mystery. Lots of work has been carried out to investigate the properties of shear bands, including structure dilatation [2, 3], diffusivity [4], and strength [3]. Since the thickness of shear bands is about few tens of nanometers [4], transmission electron microscope (TEM) is often used to study the morphology, nanoscale defects $[5,6]$, and nanocrystalline phases [7-10] in the shear bands. However, conventional TEM sample preparation methods cannot avoid some artifacts that induced by ion impinging damage or chemical thinning. The artifacts introduced during sample preparation therefore may cover up the original structure of shear bands. The nanocrystalline phase formation during the plastic deformation process has been noticed, but the exact reason of crystalline phase formation is still under debate
[9]. In this paper, to avoid any possible artifact, shear bands were introduced in the Pd-Cu-Si MG after the TEM sample preparation. Nanocrystalline phases are identified within the prepared shear bands, which may provide some hints to understand the different mechanisms of crystalline phase formation.

\section{Materials and Methods}

The ingot of the nominal $\mathrm{Pd}_{77.5} \mathrm{Cu}_{6} \mathrm{Si}_{16.5}$ metallic glass was prealloyed by high frequency induction melting the mixtures of high purity Pd (99.5\%), Cu (99.99\%), and Si (99.99\%) under argon pressure in a quartz tube and then purified three times in $\mathrm{B}_{2} \mathrm{O}_{3}$ fluxing medium under a vacuum of $10^{-2} \mathrm{~Pa}$ followed by water quenching. A cylindrical sample with $6 \mathrm{~mm}$ in diameter was obtained. The as-prepared samples were examined by X-ray diffraction (XRD) technique using a Japan Rigaku D/max-RB XRD spectrometry. The specimens of $2 \times 2 \times 4 \mathrm{~mm}^{3}$ were cut from the middle part of as-cast $\mathrm{Pd}_{77.5} \mathrm{Cu}_{6} \mathrm{Si}_{16.5}$ and used for room temperature compression tests on a WDW-50 testing machine at a strain rate of $4 \times 10^{-4} \mathrm{~m} \cdot \mathrm{s}^{-1}$. Samples with a diameter of $3 \mathrm{~mm}$ were cut from the ingot for TEM analysis. After mechanical thinning of the sample for TEM examination, the sample was subject to twin-jet electropolishing until a hole appeared. The electropolishing solution is a mixture of 77 vol. $\% \mathrm{CH}_{3} \mathrm{COOH}$ 


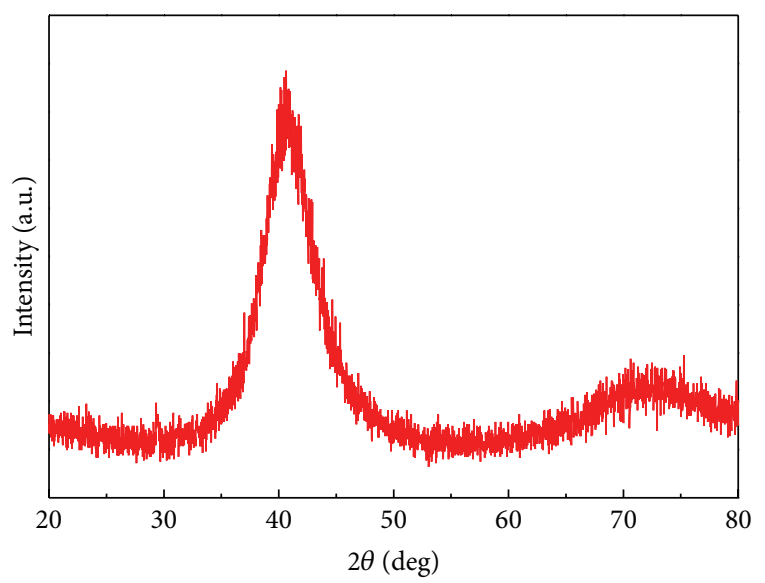

FigURE 1: X-ray diffraction spectrum of as-prepared $\mathrm{Pd}_{77.5} \mathrm{Cu}_{6} \mathrm{Si}_{16.5}$ metallic glass. Only two broad peaks could be observed indicating a glassy nature of $\mathrm{Pd}_{77.5} \mathrm{Cu}_{6} \mathrm{Si}_{16.5}$ metallic glass.

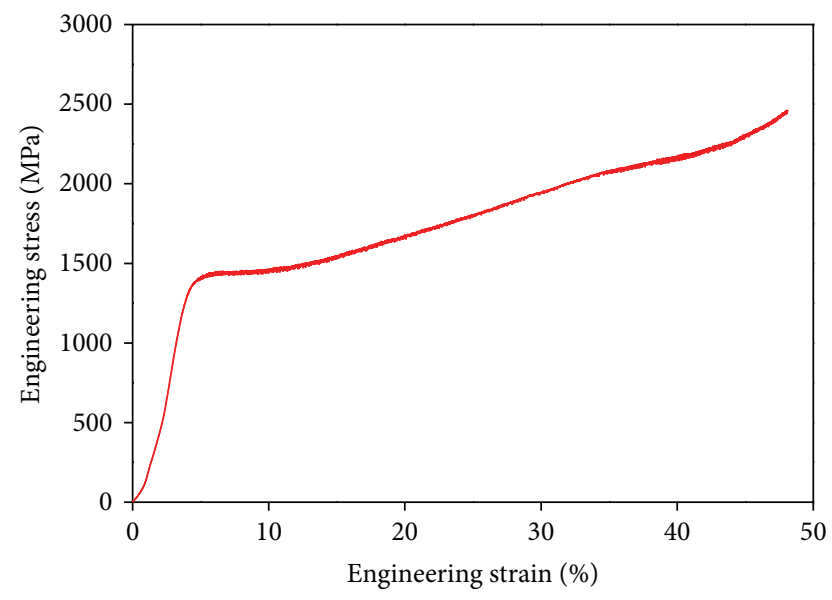

FIgURE 2: Engineering stress-strain curve for $\mathrm{Pd}_{77.5} \mathrm{Cu}_{6} \mathrm{Si}_{16.5}$ metallic glass.

plus 23 vol. $\% \mathrm{HClO}_{4}$. To avoid the possible artifacts in the subsequent observation of shear bands, which may arise from preferred thinning by either chemical polishing or ion etching during TEM sample preparation, the shear bands studied in this paper were created after TEM sample preparation by indentation near the transparent part of the sample. By doing so, a few shear bands that are suitable for TEM analysis were obtained. The TEM inspection was performed on an FEI Tecnai F20 G2 operated at $200 \mathrm{kV}$ and integrated with an FEI Eagle 2 K CCD.

\section{Results and Discussion}

Figure 1 showed the XRD spectrum of the as-prepared $\mathrm{Pd}_{77.5} \mathrm{Cu}_{6} \mathrm{Si}_{16.5}$ metallic glass. Besides the two broad peaks there, no sharp peak could be detected on the XRD spectrum in the 2-theta range from 20 to 80 degree, indicating a glassy nature of the as-prepared $\mathrm{Pd}_{77.5} \mathrm{Cu}_{6} \mathrm{Si}_{16.5}$ metallic glass. Figure 2 showed the engineering stress-strain curve of

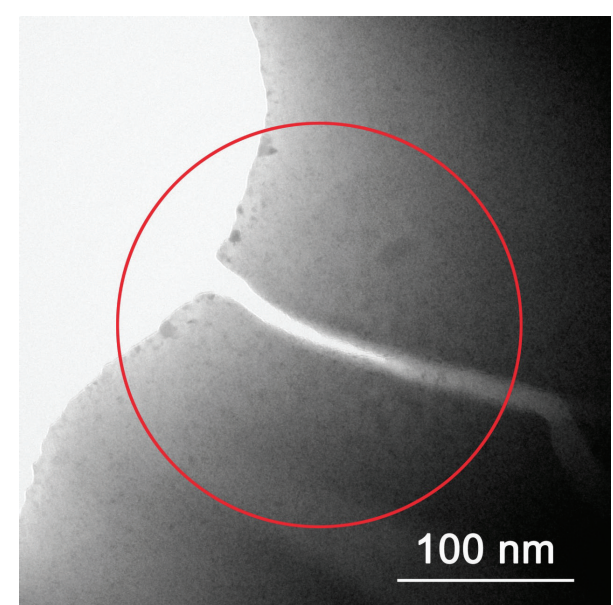

FIGURE 3: TEM bright field image of the region containing a clear shear band. The red circle indicated a region used for later electron diffraction.

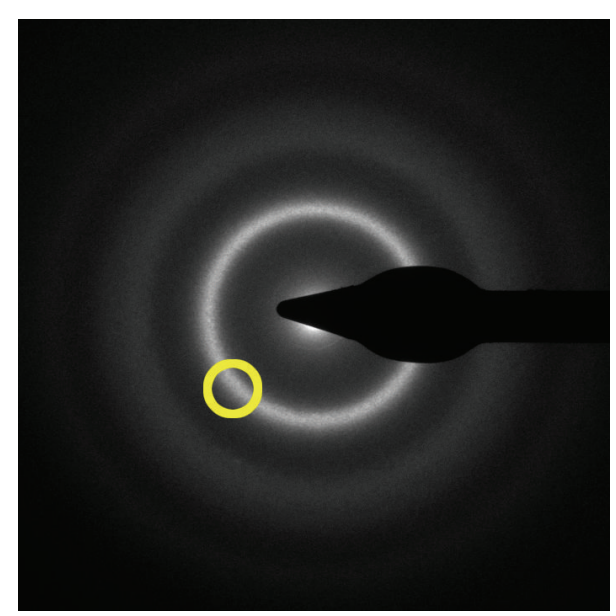

FIGURE 4: Selected area electron diffraction pattern from the region marked with red circle in Figure 2. The two diffuse halos show the glassy nature of as-prepared $\mathrm{Pd}_{77.5} \mathrm{Cu}_{6} \mathrm{Si}_{16.5}$ metallic glass in microscale. The part of inner halo marked by yellow circle was used for later TEM dark field imaging.

the as-prepared $\mathrm{Pd}_{77.5} \mathrm{Cu}_{6} \mathrm{Si}_{16.5}$ metallic glass. The yield strength of $\mathrm{Pd}_{77.5} \mathrm{Cu}_{6} \mathrm{Si}_{16.5}$ was about $1300 \mathrm{MPa}$, and the engineering strain was up to $40 \%$, showing excellent plastic deformation ability. Figure 3 showed a bright field image of the created shear band in $\mathrm{Pd}_{77.5} \mathrm{Cu}_{6} \mathrm{Si}_{16.5}$ metallic glass. From Figure 3, a clear shear band with a thickness of $13 \mathrm{~nm}$ could be noticed, and a microcrack could also be observed at one end of the shear band. A diffraction pattern that covered the region marked with a red circle in Figure 3 was shown in Figure 4. No clear diffraction spots could be noticed, except two halos in Figure 4, suggesting a glass nature of the asprepared sample, which was also consistent with our XRD result. By using a part of inner halo ring indicated by a yellow circle in Figure 4, a dark field image of TEM was shown in Figure 5. From the dark field image of TEM in Figure 5, a few bright parts that suggested the existence of nanocrystalline 


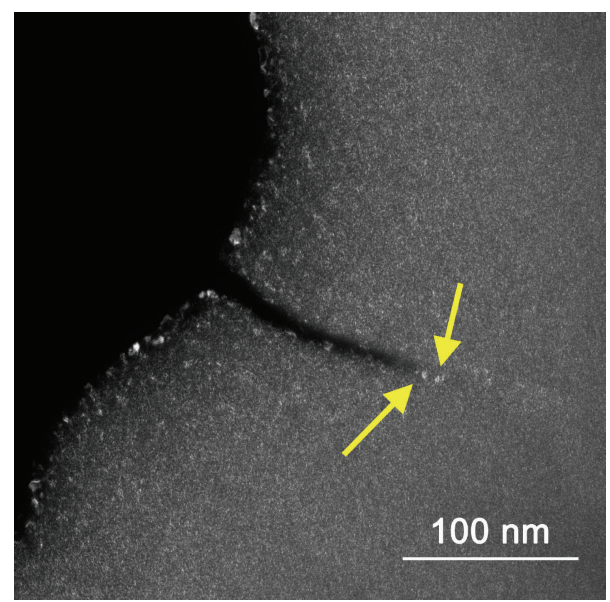

FIGURE 5: TEM dark field image of as-prepared $\mathrm{Pd}_{77.5} \mathrm{Cu}_{6} \mathrm{Si}_{16.5}$ metallic glass around a shear band. The bright parts indicated by the arrows within the shear band suggest the existence of crystalline phase formation.

phases could be noticed inside the shear band, at the edge of the crack and at the edge of the TEM sample. In addition, clear lattice fringes from high-resolution TEM results were also noticed for the nanocrystalline phases inside shear bands, which were not presented here. The nanocrystalline phase formation may have different originations at different parts in the sample. In this paper, we would like to focus only on the nanocrystalline phases formation inside shear bands.

Although crystalline phases inside shear bands have been reported [7-10] and studied by TEM, improper TEM sample preparation techniques may introduce artifacts, such as nanocrystalline phases, and it was also mentioned recently that some of crystalline phases observed in the deformed region were not because they were deformed but because they were thin [11]. In this paper, since the shear bands were created after TEM sample preparation, the deformed region was off artifacts and the observed crystalline phases were undoubtedly formed during the deformation. To explain the formation of the observed crystalline phases in shear bands, both the thermodynamic and the kinetic factors should be considered. Since the temperature can easily induce the crystallization transformation from MGs, temperature effect was proposed as an apparent reason for the crystallization formation inside shear bands. Although significant temperature rise has been noticed around shear off on some metallic glasses [12], it was also suggested that for a limited shear off, the temperature rise was not that important [13]. For the shear band that we created with only $13 \mathrm{~nm}$ in thickness on TEM thin foil sample, the shear off of the shear bands would be very limited and the shear band could be regarded as a cold one, where the thermal effect could be neglected. Another possible reason accounts for the crystalline phase formation is the mechanical deformation-induced crystallization. It was believed that a structure dilatation occurred inside shear bands during the plastic deformation [2]. Compared to the matrix region, such loosely packed regions inside shear bands would increase the mobility of local atoms [4] and would provide more possibility for structure relaxation and therefore crystalline phase formation. Since the formed crystalline phases might interact with shear bands by local stress relaxation, the crystalline phases would affect the overall mechanical properties of MGs. The existence of crystalline phases inside shear bands was often considered as an indication of having better plasticity in MGs $[8,14,15]$. However, it depends on when the crystalline phases form. If the crystalline phases form instantly while deformation, the local stress around the newly formed crystalline phase would reduce and might not be enough to support further shear band propagation. When the external load further increases, other sites may reach the critical local stress and initial another new shear band. Since the plasticity of metallic glasses is often related to the observed multiple shear bands formation, the overall plasticity would be improved if crystalline phases formed instantly while deformation. If the crystalline phases form a while after the formation of shear bands, they would have little effect on blocking the current shear band. Then the overall plasticity might not be improved, although crystalline phases were noticed. Since the $\mathrm{Pd}_{77.5} \mathrm{Cu}_{6} \mathrm{Si}_{16.5}$ MG has demonstrated excellent plastic deformation ability, we believed that the crystalline phases we observed were formed instantly as the plastic deformation proceeded. As to the preferred orientation and possible defects of the crystalline phases in the shear bands of Pd77.5Cu6Si16.5 MG, further investigations are still carrying on.

\section{Conclusions}

In summary, the $\mathrm{Pd}_{77.5} \mathrm{Cu}_{6} \mathrm{Si}_{16.5}$ metallic glass was prepared and shear bands were created by indentation after TEM sample preparation to avoid possible prefer thinning. The dark field image of TEM showed the existence of nanocrystalline phases formation inside shear bands, where they were mainly induced by mechanical deformation but not by the thermal effect. The excellent plastic deformation ability of $\mathrm{Pd}_{77.5} \mathrm{Cu}_{6} \mathrm{Si}_{16.5}$ metallic glass suggested that the crystalline phases were formed instantly while deformation and then retarded the shear band propagation.

\section{Conflict of Interests}

The authors declare that there is no conflict of interests regarding the publication of this paper.

\section{Acknowledgments}

This work was supported by the National Science Foundation of China (Grant no. 51101090), the Beijing Higher Education Young Elite Teacher Project (Grand no. YETP0121), and the Scientific Research Foundation for Returned Scholars, Ministry of Education of China.

\section{References}

[1] M. F. Ashby and A. L. Greer, "Metallic glasses as structural materials," Scripta Materialia, vol. 54, no. 3, pp. 321-326, 2006. 
[2] P. E. Donovan and W. M. Stobbs, "The structure of shear bands in metallic glasses," Acta Metallurgica, vol. 29, no. 8, pp. 14191436, 1981.

[3] J. Pan, Q. Chen, L. Liu, and Y. Li, "Softening and dilatation in a single shear band," Acta Materialia, vol. 59, no. 13, pp. 5146-5158, 2011.

[4] J. Bokeloh, S. V. Divinski, G. Reglitz, and G. Wilde, "Tracer measurements of atomic diffusion inside shear bands of a bulk metallic glass," Physical Review Letters, vol. 107, Article ID 235503, 2011.

[5] J. Li, Z. L. Wang, and T. C. Hufnagel, "Characterization of nanometer-scale defects in metallic glasses by quantitative high-resolution transmission electron microscopy," Physical Review B, vol. 65, no. 14, Article ID 144201, pp. 1442011-1442016, 2002.

[6] J. Li, F. Spaepen, and T. C. Hufnagel, "Nanometre-scale defects in shear bands in a metallic glass," Philosophical Magazine A, vol. 82, no. 13, pp. 2623-2630, 2002.

[7] G. Wilde and H. Rösner, "Nanocrystallization in a shear band: an in situ investigation," Applied Physics Letters, vol. 98, no. 25, Article ID 251904, 2011.

[8] M. Chen, A. Inoue, W. Zhang, and T. Sakurai, "Extraordinary plasticity of ductile bulk metallic glasses," Physical Review Letters, vol. 96, no. 24, Article ID 245502, 2006.

[9] G. Kumar, T. Ohkubo, T. Mukai, and K. Hono, "Plasticity and microstructure of $\mathrm{Zr}$-Cu-Al bulk metallic glasses," Scripta Materialia, vol. 57, no. 2, pp. 173-176, 2007.

[10] K. Wang, T. Fujita, Y. Q. Zeng, N. Nishiyama, A. Inoue, and M. W. Chen, "Micromechanisms of serrated flow in a $\mathrm{Ni}_{50} \mathrm{Pd}_{30} \mathrm{P}_{20}$ bulk metallic glass with a large compression plasticity," Acta Materialia, vol. 56, no. 12, pp. 2834-2842, 2008.

[11] A. L. Greer, Y. Q. Cheng, and E. Ma, "Shear bands in metallic glasses," Materials Science and Engineering R, vol. 74, pp. 71-132, 2013.

[12] J. J. Lewandowski and A. L. Greer, "Temperature rise at shear bands in metallic glasses," Nature Materials, vol. 5, no. 1, pp. 1518, 2006.

[13] D. B. Miracle, A. Concustell, Y. Zhang, A. R. Yavari, and A. L. Greer, "Shear bands in metallic glasses: size effects on thermal profiles," Acta Materialia, vol. 59, no. 7, pp. 2831-2840, 2011.

[14] K. Hajlaoui, A. R. Yavari, B. Doisneau et al., "Shear delocalization and crack blunting of a metallic glass containing nanoparticles: in situ deformation in TEM analysis," Scripta Materialia, vol. 54, no. 11, pp. 1829-1834, 2006.

[15] S.-W. Lee, M.-Y. Huh, E. Fleury, and J.-C. Lee, "Crystallizationinduced plasticity of $\mathrm{Cu}-\mathrm{Zr}$ containing bulk amorphous alloys," Acta Materialia, vol. 54, no. 2, pp. 349-355, 2006. 

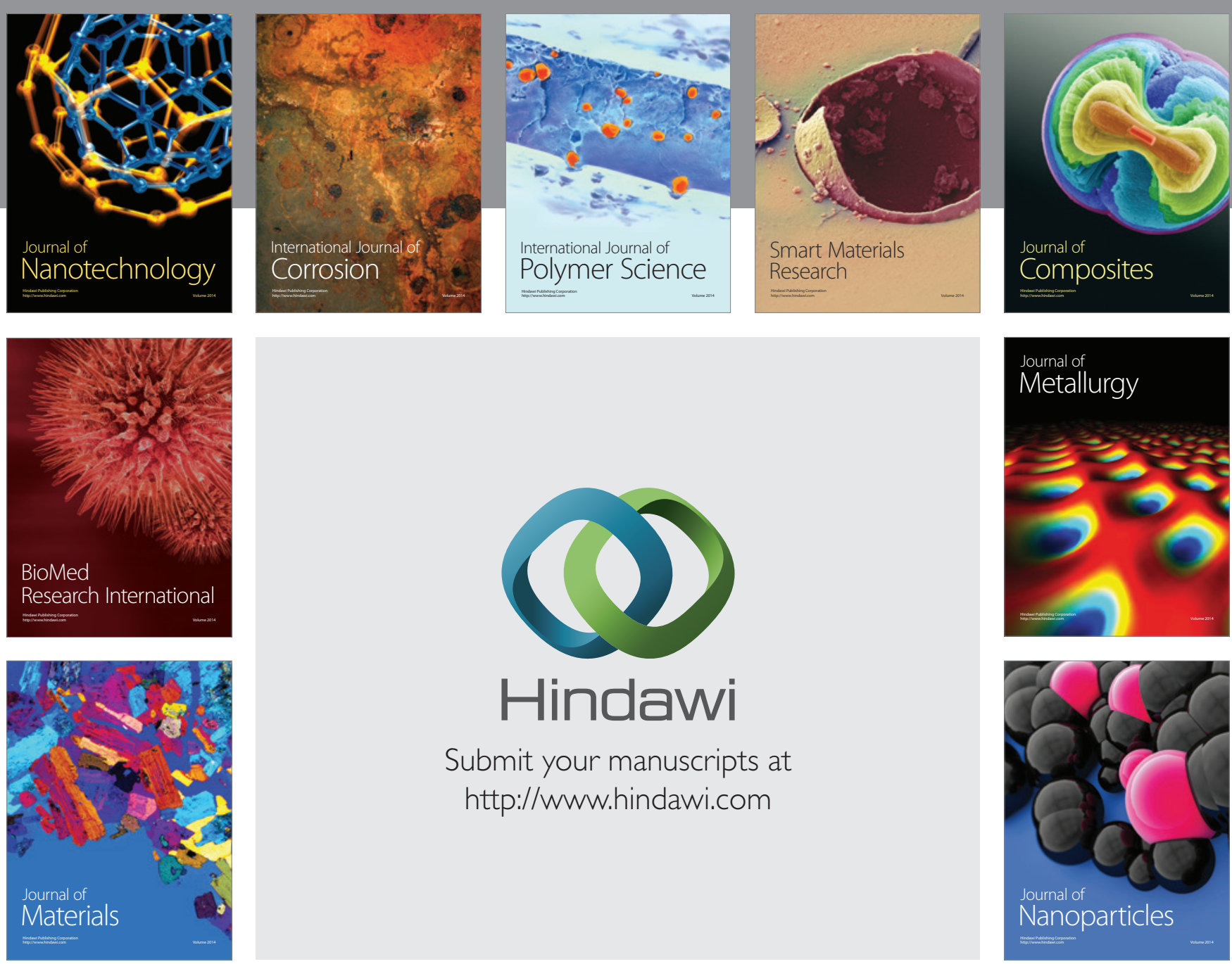

Submit your manuscripts at http://www.hindawi.com
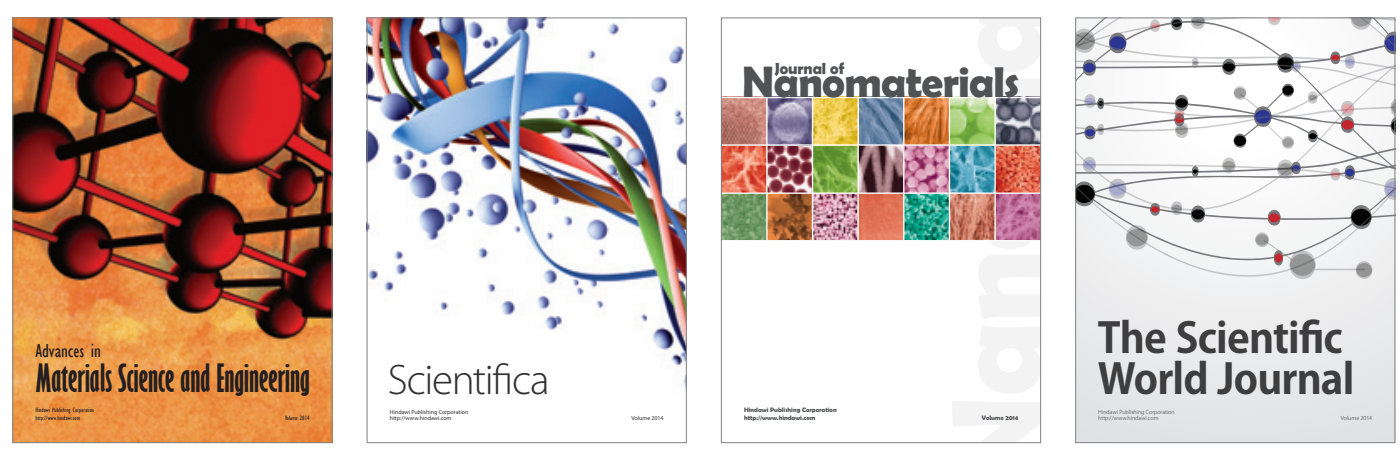

\section{The Scientific World Journal}
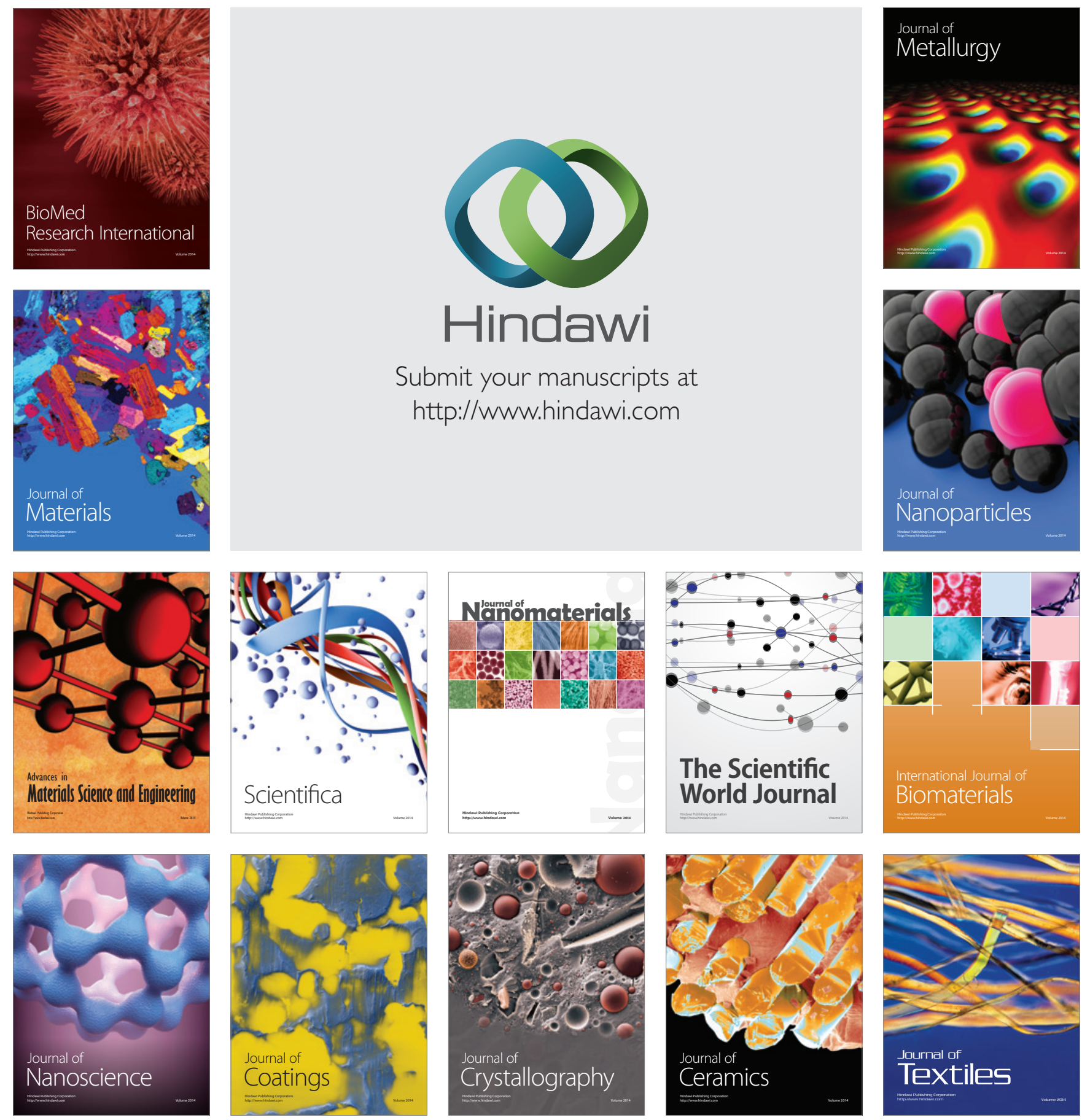